\title{
Visceral artery aneurysms
}

\section{Aneurismas viscerais}

Sergio Silveira leal Meirelles, tCBC-RJ'.

he Visceral Artery Aneurysms (VAA), although
considered rare for a long time, actually are not so unusual. Their incidence ranges from 1.5 to $3 \%$ in the literature and consists of segmental dilatation of the visceral branches of the abdominal aorta. By not presenting with specific characteristic symptoms, they have great clinical importance, since about $22 \%$ of them will manifest as a surgical emergency.

Classically, their diagnoses were made during laparotomy for treatment of hemorrhagic acute abdomen. Presently, modern imaging tests allow early diagnosis of visceral aneurysms.

This issue of the Journal of the Brazilian College of Surgeons (CBC) brings three such cases. The splenic artery aneurysm is the most prevalent and may be present in up to $10.4 \%$ of autopsies in patients over 60 years. It is most often asymptomatic, and rupture may be the first symptom. Especially prone to rupture are the aneurysms in pregnant women in the 3rd trimester. In the report, the aneurysm is diagnosed during a magnetic resonance angiography of the abdominal aorta, during the investigation of cervical lymphadenopathy in a 51-year-old patient. Although asymptomatic, surgery was indicated since the aneurysm had a diameter of $2.5 \mathrm{~cm}$. It was possible to deal with that case with an elective splenectomy.

The hepatic artery aneurysm is the third in frequency among visceral aneurysms. The location is extrahepatic in $80 \%$ of cases and intrahepatic in the remaining $20 \%$. Most display a saccular form. The common hepatic artery is the most affected, followed by its right branch, which is the site of the reported aneurysm. In this report, the patient was symptomatic, with abdominal pain and anemia, which made the casemore complex. The diagnosis was only possible with an abdominal computed tomography (CT) and surgery consisted in the ligation of the right hepatic artery. In high-risk patients, as reported, the surgeon would also have the option of using an endovascular technique for selective embolization of that artery.

The third case is of a celiac artery aneurysm rupture caused by Behçet's disease, actually an extremely rare case. Celiac trunk aneurysms account for $4 \%$ of visceral aneurysms, the vast majority of patients being asymptomatic. Many are detected on imaging studies performed to investigate other diseases. In such cases, as the authors point out, open surgery with resection of the aneurysm and vascular reconstruction is a good option. One can also employ endovascular treatment with stent implantation or coil embolization. In this case, due to theemergency, it was decided to resect theaneurysm and suture the aorta, which proved to be a good option when facing such a challenge, the patient presenting with a successful recovery after 40 days of hospitalization.

Currently, most VAA can be treated by endovascular methods. The indications are the same as surgery: saccular form, symptoms of expansion or rupture. All pseudoaneurysms should be treated. According to Arno von Ristow, although there are authors who recommend the treatment of asymptomatic truncal VAA from $15 \mathrm{~mm}$ diameter on, $20 \mathrm{~mm}$ is the accepted limit;andit is a consensus that non-saccular asymptomatic saccular VAA that have three times the original diameter of the normal native artery require treatment.

1 - Servidores do Estado Federal Hospital, Rio de Janeiro, RJ, Brazil. 